\title{
Chronik der Rundfunkentwicklung in Deutschland 1999
}

\section{Christiane Matzen / Anja Herzog}

1. Allgemeines

1.1 Medienentwicklung europäisch/international

1.2 Medienpolitik Bund/Länder

1.3 Medienrecht

1.4 Medienkonzerne

1.5 Marktanteile/Werbemarkt
2. Öffentlich-rechtlicher Rundfunk gesamt

2.1 Einzelne Anstalten

3. Privater Rundfunk

3.1 Landesmedienanstalten

3.2 Private Hörfunkveranstalter

3.3 Private Fernsebveranstalter

\section{Allgemeines}

\subsection{Medienentwicklung europäisch / international}

Die EU-Kommission weist die Beschwerden des Verbandes Privater Rundfunk und Telekommunikation (VPRT) und einiger privater TV-Anbieter gegen die öffentlich-rechtlichen Spartenkanäle Der Kinderkanal und Phoenix am 8. März zurück. ${ }^{1}$ Die wettbewerbsrechtlichen Vorschriften des EG-Vertrages seien nicht verletzt, da ARD und ZDF auf dem Werbemarkt mit zusammen nur etwa 10 Prozent keine marktbeherrschende Stellung einnähmen und die öffentlich-rechtlichen Spartenkanäle zudem werbefrei seien.

Am 23. Juli nimmt die Europäische Kommission eine Richtlinie ${ }^{2}$ an, die die Telekommunikationsunternehmen in den Mitgliedsländern darauf verpflichtet, Kabelfernsehnetze in rechtlich selbstständigen Einheiten zu betreiben. Die Kommission möchte auf diese Weise den Wettbewerb und Innovation im Bereich des Kabelfernsehens sowie bei den örtlichen Fernmeldenetzen und beim breitbandigen Internet-Zugang fördern.

Die Generalversammlung des Gremiums für Digital Video Broadcasting (DVB) einigt sich am 10. November in Genf auf einen europäischen Multimedia-Standard, die Multimedia Home Platform (MHP). Diese umfasst u. a. eine offene Schnittstelle API (Application Programming Interface) und das Common Interface CI, die auf der Programmiersprache Java basieren. Mit diesem Standard kann eine einheitliche Software zur Steuerung der Empfangsgeräte wie Set-Top-Boxen und integrierte Endgeräte entwickelt werden.

\subsection{Medienpolitik Bund / Länder}

Die Ministerpräsidenten der Länder verabschieden am 24. Juni den 4. Rundfunkänderungsstaatsvertrag ${ }^{3}$, der - nach Ratifizierung durch die Landesparlamente-zum 1. April 2000 in Kraft treten soll. Die Novelle setzt vor allem die Regelungen der EU-Fernsehrichtlinie vom Juni 1997 in deutsches Recht um.

1 Abdruck der Entscheidung in epd medien Nr. 29/1999 und Funkkorrespondenz Nr. 16/1999.

2 Abdruck der Kabel-Richtlinie 1999/64/EG, Abl. L 175/39 - 42 vom 10. Juli in epd medien Nr. 56/1999.

3 Abgedruckt in epd medien 36/1999 und Funkkorrespondenz Nr. 52/1999. 
Die Staatskanzleien von Rheinland-Pfalz und Baden-Württemberg vereinbaren am 8. Juli, dass der Südwestrundfunk (SWR) sein bislang nur digital und per Internet empfangbares Jugendradio „Das Ding“ ab April 2000 auch terrestrisch in den Ballungsräumen der beiden Bundesländer verbreiten darf. Werbung und Sponsoring dürfen nicht zur Finanzierung dienen. Durch die UKW-Zulassung des Jugendprogramms hat der SWR dann faktisch fünf Hörfunkprogramme, obwohl im SWR-Staatsvertrag insgesamt nur vier Radioprogramme vorgesehen sind.

Auf ihrer Jahreskonferenz am 12. November in Bremen beschließen die Ministerpräsidenten eine Neuregelung des ARD-Finanzausgleichs und folgen damit den Empfehlungen der Rundfunkkommission der Länder vom 15. Oktober. Der ARD-Finanzausgleich soll demzufolge ab dem 1.1.2001 von 186 Mio. (entspricht 1,9 \% des ARD-Gebührenanteils) auf 160 Mio. DM und bis 2006 weiter linear auf 1 Prozent des ARD-Gebührenanteils reduziert werden. Des Weiteren sprechen sich die Ministerpräsidenten für die Einführung eines gewichteten Stimmrechts der Landesrundfunkanstalten in der ARD aus, so dass große Anstalten wie der WDR mehr Stimmengewicht erhalten als kleine, wie z. B. Radio Bremen. Außerdem fordern die Ministerpräsidenten die Bundesregierung auf, „ein schlüssiges und finanziell tragbares Konzept“ für den Auslandsrundfunk zu entwickeln.

\subsection{Medienrecht}

Das Bundesverfassungsgericht lehnt es am 15. Januar ab, die Verfassungsbeschwerde von Radio Bremen gegen Übergangsvorschriften im Gesetz zur Änderung des Radio-Bremen-Gesetzes vom 27.10.1998 ${ }^{4}$ zur Entscheidung anzunehmen. ${ }^{5}$ Das Gesetz sieht unter anderem eine neue Leitungsstruktur mit stärkerer Verantwortung des Intendanten vor.

Am 13. April tritt die Novelle zum Gesetz über den Ostdeutschen Rundfunk Brandenburg $(\mathrm{ORB})^{6}$ in Kraft. Diese verpflichtet den ORB weiterhin zur Ausstrahlung von Wahlwerbung, nachdem der Sender zuvor versucht hatte, sich von der Pflicht zur Sendung von Wahlspots befreien zu lassen.

Der Landtag von Baden-Württemberg verabschiedet am 14. Juli ein neues Landesmediengesetz für den Privatfunk ${ }^{7}$, das am 28. Juli 1999 in Kraft tritt.

Die Verfassungsbeschwerde einer Hotelbetreiberin, die sich gegen die Gebührenpflicht gerichtet hatte, wird von der Ersten Kammer des Bundesverfassungsgerichts (BVerfG) am 28. September nicht zur Entscheidung angenommen. Die Hotelbetreiberin hatte argumentiert, durch die Gebührenpflicht sei ein ungehinderter Zugang zu den Informationsangeboten privater Veranstalter und ausländischer Programme nicht möglich und somit Art. 5 I 1 des Grundgesetzes verletzt. Dem BVerfG zufolge beinhaltet der Artikel 5 GG aber keine Garantie für kostenlose Informationen.

Am 13. Oktober verabschiedet der Landtag von Schleswig-Holstein ein novelliertes Landesrundfunkgesetz ${ }^{8}$, das am 29. Oktober in Kraft tritt.

4 Gesetz über die Errichtung und die Aufgaben einer Anstalt des öffentlichen Rechts - Radio Bremen - in der Fassung vom 29. Oktober 1998, abgedruckt in Funkkorrespondenz, Nr. 45/1998, Beilage.

5 Abdruck der Entscheidung des BVerfG in epd medien Nr. 8/1999.

6 Abgedruckt in Funkkorrespondenz Nr. 18 - 19/1999, Beilage.

7 Abgedruckt in Funkkorrespondenz Nr. 32/1999, Beilage.

8 Rundfunkgesetz für das Land Schleswig-Holstein, novellierte Fassung abgeruckt in epd medien Nr. 90/1999. 
Der Bundesgerichtshof (BGH) in Karlsruhe entscheidet am 21. Oktober, dass der private Fernsehveranstalter Kabel 1 in seinem Senderlogo (wie ursprünglich) die Ziffer „Eins“ verwenden darf. Es besteht laut Urteil keine Verwechslungsgefahr mit der „Eins“ des ersten Fernsehprogramms der ARD. Damit hebt der BGH das anders lautende Urteil des Kölner Oberlandesgerichts vom 9. April 1997 auf.

Die Klage der beiden privaten Fernsehsender SAT.1 und Vox gegen das als „Fernsehfee" bezeichnete Gerät zur Ausblendung von Werbung aus ihrem Programm wird vom Berliner Kammergericht am 22. Oktober abgewiesen. Das von der TC Unterhaltungselektronik AG vertriebene Gerät greife nicht in die Rundfunkfreiheit ein und bedrohe auch nicht die Existenz von privaten Fernsehsendern. Damit heben die Richter eine Einstweilige Verfügung des Berliner Landgerichts von Ende Mai 1999 auf, die es der TC Unterhaltungselektronik untersagte, bundesweit Signale auszustrahlen, mit denen in den Programmen von SAT.1 und Vox die Werbung automatisch ausgeblendet wird.

Der Europäische Gerichtshof (EuGH) entscheidet mit seinem Urteil vom 28. Oktober, dass die EG-Fernsehrichtlinie das so genannte Brutto-Prinzip bei der Berechnung von Werbeunterbrechungen vorsieht. Das bedeutet, dass die Sender bei der Festlegung der Spielfilmlänge die Werbespots hinzurechnen dürfen und sich so die Werbezeit erhöht, die auf Grundlage der Spielfilmlänge errechnet wird. In Deutschland darf ein Spiel- oder Fernsehfilm für jeden vollen Zeitraum von 45 Minuten einmal durch Werbung unterbrochen werden; eine weitere Unterbrechung ist zulässig, wenn die Sendedauer um mindestens 20 Minuten über zwei oder mehrere volle 45 Minutenzeiträume hinausgeht.

Beim Mainzer Landgericht reichen drei deutsche Vergnügungsparks am 4. November Klage gegen den geplanten ZDF-Medienpark ein. Die drei Parks wollen dem Sender gerichtlich untersagen lassen, „einen ZDF-Erlebnispark zu entwickeln und zu betreiben, soweit dieser ,Fahrgeschäfte“ und/oder ,Simulatoren' enthält.“ Nach ihrer Meinung gestatte das deutsche Rundfunkrecht ein solches Projekt nicht und verstoße zudem gegen das Gesetz gegen unlauteren Wettbewerb.

\subsection{Medienkonzerne}

Die Kirch-Gruppe gibt sich mit Jahresbeginn eine neue Struktur mit drei Dachgesellschaften: die Kirch Media KGaA (früher: TaurusFilm GmbH \& Co KG) für die Geschäftsfelder Lizenzhandel, werbefinanziertes Fernsehen, Produktion und Filmbearbeitung, die Kirch PayTV KGaA (früher: PayCo Holding GmbH \& Co KG) sowie die Taurus-Beteiligungs GmbH \& Co KG, der u. a. die 40\%-Beteiligung am Axel-Springer-Verlag und die Decoderfirma BetaResearch zugeordnet sind. Durch die gewählte Rechtsform der „Kommanditgesellschaften auf Aktien“ (KGaA) könnten die beiden erstgenannten Dachgesellschaften dem Kapitalmarkt geöffnet werden.

Das Kirch-Tochterunternehmen BetaResearch präsentiert am 12. Januar für ihren digitalen Empfangsdecoder d-Box eine offene Programmschnittstelle (API) mit Namen Betanova. Auch andere Anbieter digitaler TV-Plattformen können die d-Box nun für ihre Angebote, wie elektronische Programmführer, Spiele und interaktive Dienste, nutzen. Künftig soll neben der bisher einzigen Herstellerfirma Nokia auch die niederländische Philips-Tochter Digital Video System in die Produktion der d-Box einsteigen.

Die Kommission zur Ermittlung der Konzentration im Medienbereich (KEK) beschließt am 26. Januar, dass die Vorgaben im Rundfunkstaatsvertrag zur Meinungsvielfalt der Zulassung der digitalen Pay-TV-Programme von Premiere nicht entgegen stehen. Die Aufstockung der Beteiligung von Thomas Kirch am Grundkapital des Fern- 
sehsenders Pro Sieben von 24,5 auf 58,4 Prozent sei ebenfalls unbedenklich, da die KirchGruppe insgesamt nicht den im Rundfunkstaatsvertrag als Grenze benannten Zuschaueranteil von 30 Prozent erreiche. Am 21. September schließlich wird auch die marktbeherrschende Stellung der Kirch-Gruppe auf dem „vorgelagerten medienrelevanten Markt der Zugangsvermittlung “ zum digitalen Pay-TV für unbedenklich erklärt. Die gesellschaftsrechtlichen Veränderungen bei DF1 und Premiere, die im Oktober zu Premiere World fusionieren, werden danach keine „vorherrschende Meinungsmacht“ ergeben.

Die Pro Sieben Media AG kündigt am 2. Februar an, spätestens im Januar 2000 einen eigenen bundesweiten 24-Stunden-Nachrichtensender mit dem Arbeitstitel N24 mit besonderem Schwerpunkt auf der Wirtschaftsberichterstattung zu starten. Der Pro Sieben-Vorstandsvorsitzende Georg Kofler erklärt, dass N24 Teil einer Gesamtstrategie sei, die eine „längerfristige und vitale strategische Weichenstellung“ bedeute. Binnen 18 Monaten wolle die Pro Sieben Media AG „zum führenden Anbieter von audiovisueller Information" werden.

Unter dem Namen Free Universe Network (FUN) gründen Unternehmen der Kommunikationstechnik und der Medienwirtschaft sowie mehrere Rundfunkveranstalter, darunter Mitglieder der ARD, am 12. Februar eine unabhängige Digitalplattform. FUN will für Deutschland einen unabhängigen digitalen TV-Standard entwickeln und sich für Vielfalt, offenen Zugang, Meinungsfreiheit und Wettbewerb auch beim digitalen Fernsehen einsetzen.

Nachdem die Kirch-Gruppe am 25. März bekannt gegeben hat, dass sie beim Pay-TVSender Premiere die Anteile der Bertelsmann-Tochter CLT-Ufa übernommen habe, kündigt sie fünf Tage später an, die digitalen Fernsehaktivitäten von Premiere und DF1 bündeln und den Sitz von Premiere von Hamburg nach München verlegen zu wollen. 95 Prozent von Premiere gehören nun der Kirch-Gruppe, 5 Prozent behält der Bertelsmann-Konzern, um sich weiterhin den Zugang seines internationalen Rechtehandels zum deutschen Pay-TV-Markt zu sichern; der französische Pay-TV-Anbieter Canal plus scheidet gänzlich aus. Das Bundeskartellamt stimmt der Anteilsübernahme am 14. April zu.

Am 8. Oktober teilen die Kirch-Gruppe und die Pro Sieben Media AG mit, dass die Kirch-Gruppe Mehrheitseigner der Pro Sieben Media AG wird. Der Hauptaktionär der stimmberechtigten Pro-Sieben-Stammaktien, Thomas Kirch, überträgt seinen Anteil $(58,4 \%)$ auf die Kirch Media GmbH \& Co. KGaA seines Vaters Leo Kirch. Thomas Kirch erhält dafür zwischen 7 und 8 Prozent der Kirch Media KGaA. Der Zuschauermarktanteil der vier in Kirch-Besitz befindlichen Free-TV-Sender (SAT.1, DSF, Pro Sieben und Kabel 1) beträgt nun zusammen etwas über 26 Prozent und bleibt somit unter der im Rundfunkstaatsvertrag vorgesehenen 30-Prozent-Grenze.

In einer Presseerklärung vom 26. Oktober teilt die Kirch-Gruppe mit, dass das USInvestmentfondsunternehmen Capital Research and Management Funds neuer Gesellschafter der Kirch Media KGaA wird. Der Investmentfonds wird für 507,8 Mio. DM 3,5 Prozent der Kirch Media KGaA übernehmen. Mit 87,5 Prozent bleibt die Mehrheit der Anteile bei Kirch Media.

Am 24. November wird bekannt, dass die Kirch-Gruppe für die digitale Pay-TVPlattform Premiere World neue Kredite in Höhe von 3 Mrd. DM erhalten wird. Die Kredite müssen bis Ende 2000 zurückgezahlt werden. Kreditgeber sind die US-Bank Chase Manhattan und ein deutsches Bankenkonsortium.

Am 6. Dezember teilt die Kirch-Gruppe mit, Verträge mit dem größten Pay-TV-Veranstalter, dem von Rupert Murdoch beherrschten British Sky Broadcasting (BSkyB) abgeschlossen zu haben. Murdoch beteiligt sich an der Kirch Pay-TV GmbH und Co. 
KGaA (Premiere World) mit 2,9 Mrd. DM (24 \%) und erhält gleichzeitig weit reichende Einspruchsrechte für Kirchs Pay-TV-Plattform.

\subsection{Marktanteile/Werbemarkt}

Die Arbeitsgemeinschaft Fernsehforschung (AGF) gibt am 9. Dezember bekannt, dass die Zusammenarbeit von öffentlich-rechtlichen und privaten Fernsehsendern bei der kontinuierlichen quantitativen Fernsehzuschauerforschung mit einem neuen Vertrag (ab 1. Januar 2000) auf unbefristete Zeit fortgeführt wird. Im Vertrag wurde eine neue Binnenstruktur der AGF festgelegt, die nicht mehr aus Einzelmitgliedschaften bestehen, sondern aus Senderfamilien gebildet wird: ARD-Familie, ZDF-Familie, Pro-Sieben-Familie, RTL-Familie und SAT.1-Familie. Hiermit verbunden, ist ein neues Finanzierungsmodell: Neben einem gleich hohen Sockelbeitrag der Senderfamilien wird es einen marktanteilsgebundenen variablen Kostenanteil geben. Mit der GfK wurde ein neuer Fünfjahresvertrag zur Ermittlung der Einschaltquoten und Marktanteile abgeschlossen. Damit verbunden ist eine Erweiterung des Fernsehpanels, darunter auch erstmals EU-Ausländerhaushalte. Nicht erfasst werden weiter die in Deutschland lebenden Ausländer aus Nicht-EU-Staaten.

Die tägliche durchschnittliche Sehdauer aller Zuschauer liegt 1999 mit 185 Minuten drei Minuten niedriger als im Vorjahr.

RTL ist 1999 beim Gesamtpublikum Sieger mit einem Marktanteil von 14,8 Prozent, gefolgt von der ARD (14,2 \%; -1,2 \% gegenüber dem Vorjahr), dem ZDF (13,2\%; $-1 \%$ gegenüber 1998), den Dritten (12,5\%), SAT.1 (10,8 \%) und ProSieben mit 8,4 Prozent. Der Marktanteil von Kabel 1 liegt 1999 bei 5,4 Prozent (+1 \% gegenüber 1998), RTL 2 kommt auf 4 Prozent, Vox und Super RTL erzielen je 2,8 Prozent, der Kinderkanal und DSF je 1,3 Prozent. Es folgen tm3 (1,0\%), 3sat (0,9\%), n-tv (0,7 \%) sowie arte und Phoenix mit je 0,3 Prozent. In der Zielgruppe der 3- bis 13-Jährigen ist Super RTL Sieger mit einem Marktanteil von 18,7 Prozent, während die Öffentlich-Rechtlichen bei den über 50-Jährigen Marktführer bleiben (ARD: $19 \%$, ZDF: 18,5 \%, die Dritten $17,4 \%)$.

Fast drei Viertel aller TV-Werbeeinnahmen gehen 1999 an drei Sender: RTL erzielt Brutto-Werbeerlöse von 3.809 Mio. DM (+4,5 \% gegenüber dem Vorjahr), SAT.1 kommt auf 3.065 Mio. DM (+1,9 \%) und ProSieben erzielt 2.843 Mio. DM (+2,8\%). RTL 2 kann seine Brutto-Werbeerlöse um 10,4 Prozent steigern (von 670 auf 740 Mio. DM). Bei den öffentlich-rechtlichen Fernsehprogrammen kommt die ARD auf Einnahmen in Höhe von 510 Mio. DM (+0,9 \%), das ZDF erzielt 413 Mio. DM (+0,8 \%).

Im Hörfunkbereich können die öffentlich-rechtlichen Radios ihre Brutto-Werbeerlöse um 6,5 \% gegenüber dem Vorjahr steigern (Spitzenreiter ist hier SWR 3 mit Einnahmen in Höhe von 111,5 Mio. DM, das entspricht einem Plus von 12,1 \% gegenüber 1998). Bei den Privatradios kann Antenne Bayern (125,2 Mio. DM; +27 \%) vor radio NRW $(113,7 ;+13,2 \%)$ den ersten Platz bei den Brutto-Werbeerlösen erringen.

Nach der am 23. Juli veröffentlichen Media-Analyse 1999 (MA '99) erreichen die 58 werbefinanzierten und werbefreien ARD-Hörfunkprogramme weiterhin mehr Hörer als die 172 werbefinanzierten privaten Radios. An einem durchschnittlichen Wochentag werden die ARD-Radioprogramme von 34,6 Mio. Erwachsenen ab 14 Jahren gehört und erzielen damit eine Hörerreichweite von 54,2 Prozent (+0,1 \% gegenüber 1998); die Privaten kommen auf einen Höreranteil von 4,2 Prozent. Beim ausschließlichen Vergleich der werbefinanzierten privaten und öffentlich-rechtlichen Wellen liegen die Marktanteile allerdings eng beieinander: Die werbefinanzierten ARD-Hörfunkprogramme er- 
reichen pro Stunde 8,07 Mio. Hörer, die privaten Radios verbessern ihre Reichweite auf 8,02 Mio. Hörer pro Stunde.

\section{2. Öffentlich-rechtlicher Rundfunk gesamt}

Die Arbeitsgruppe Deutsches Auslandsfernsehen legt am 25. Juli ihren Abschlussbericht ${ }^{9}$ vor. Die Arbeitsgruppe, die 1998 auf Beschluss der Intendanten des WDR (ARD), des ZDF und der Deutschen Welle gegründet worden war, hatte den Auftrag zu prüfen, in welcher Form und unter welchen strukturellen, rechtlichen und wirtschaftlichen Vorgaben ein deutschsprachiger Auslandskanal unter Beteiligung von $\mathrm{ARD}$ und ZDF gestaltet werden könnte. Im Abschlussbericht werden neun Strukturmodelle diskutiert und ein Modell vorgeschlagen, nach dem ARD und ZDF für das neu konzipierte Auslandsfernsehen je 38,5 Prozent und die DW 23 Prozent des Programms zuliefern.

Die ARD-Intendanten einigen sich am 24. November über eine Reform des ARDFinanzausgleichs. Künftig soll der interne Finanzausgleich (bis zum 31. Dezember 2005) auf 1 Prozent des Netto-Gebührenaufkommens reduziert werden. Damit folgt die Intendantenkonferenz dem Beschluss der Ministerpräsidenten der Bundesländer vom 12. November. Die beiden kleinsten ARD-Anstalten, Saarländischer Rundfunk (SR) und Radio Bremen (RB), sollen sich zu ihrer Entlastung mit weniger Geld als bisher an den Gemeinschaftskosten beim Film- und Sportrechtekauf für das Erste Programm beteiligen. Dagegen werden SR und RB auch weiterhin 2,5 Prozent zum ARD-Gemeinschaftsprogramm beisteuern.

Am 11. Dezember vergibt der europäische Fußballverband UEFA die Fernsehrechte an der Europameisterschaft 2004 in Portugal an die EBU (Union der Europäischen Rundfunkorganisationen). Für Deutschland werden sich ARD und ZDF die Senderechte teilen. Das Nachsehen hatten die Konkurrenten Kirch-Gruppe (SAT.1, DSF, Premiere World) und der Filmhändler Herbert Kloiber (tm3). Nach Angaben der ARD gab die Zusicherung, alle Spiele im Free-TV zu übertragen, den Ausschlag für die UEFA-Entscheidung.

Die ARD-Intendanten stimmen am 14. Dezember der Gründung einer Deutschen Mediathek, einem Museum für deutsche Rundfunkprogrammgeschichte, zu und werden dieses mit rund 900.000 DM im Jahr unterstützen. Die potenziellen Betreiber sind außerdem das Land Berlin, der französische Mischkonzern Vivendi, dem das ehemalige Defa-Gelände in Potsdam-Babelsberg gehört, RTL, SAT.1, Vox und ZDF.

$\mathrm{Ab}$ dem 29. Dezember bieten ARD und ZDF nicht mehr gemeinsam Videotext an, sondern produzieren mit „ARD Text“ und „ZDF Text“ jeweils eigene Angebote. Das ZDF hatte den gemeinsamen Vertrag Ende 1998 gekündigt, da aufgrund der gesonderten ARD-Angebote in den Dritten Programmen ein Ungleichgewicht entstanden sei.

\section{1 Öffentlich-rechtlicher Rundfunk - einzelne Anstalten}

Am 25. März setzt der Haushaltsausschuss des Deutschen Bundestages den Betriebshaushalt der Deutschen Welle (DW) für 1999 auf 601 Mio. DM fest; 1998 waren es 640 Mio. DM. Am 16. August stellt die Geschäftsführung der Deutschen Welle ein Spar-

9 Abgedruckt in epd medien Nr. 56/1999 und Funkkorrespondenz Nr. 30/1999. 
konzept vor, das einen Arbeitsplatzabbau von 745 Stellen vorsieht. Nach den Plänen der Bundesregierung soll der Etat der DW bis 2003 auf 527 Mio. DM im Betriebs- und 19 Mio. DM im Investitionsetat gekürzt werden. Der Verwaltungsrat der Deutschen Welle (DW) stimmt am 25. Oktober einem Maßnahmenpaket zum Personalabbau zu, in dem allerdings statt der ursprünglich geplanten 745 Arbeitsplätze nur noch zwischen 560 und 620 Stellen gestrichen werden sollen.

Ein Prüfgutachten des sächsischen Staatssekretärs Michael Sagurna, das die Gewerkschaften mit Blick auf die Privatisierung der Fernseh- und Hörfunktechnik des Mitteldeutschen Rundfunks (MDR) beantragt hatten, bestätigt dem Sender am 18. Mai, dass seine viel kritisierten Outsourcing-Aktivitäten nicht gesetzes- und verfassungswidrig sind. Ein vom MDR selbst in Auftrag gegebenes und am 15. Oktober veröffentlichtes Gutachten der PwC Deutsche Revision formuliert jedoch Bedenken in Bezug auf die Outsourcing-Aktivitäten des MDR. Die Wirtschaftsprüfer weisen in Bezug auf die MDR-Tochter Drefa-Holding auf Defizite im strategischen Bereich und bei der internen Kontrolle des Unternehmens MDR hin. Allgemein kritisieren die Gutachter, dass bei der „Komplexität des Beteiligungsportfolios“ kein „wirkungsvolles Controllingund Informationssystem " implantiert worden sei. Der Sächsische Rechnungshof fordert am 4. November „begrenzte Prüfrechte bei Mehrheitsbeteiligungen“, was eine Änderung des MDR-Staatsvertrags notwendig machen würde. Die Rechnungsprüfer werfen dem Sender vor, dass mit den Tochterfirmen „Vertragskonditionen und Preise intern vereinbart“ werden und so eventuell „eine verdeckte Gewinnmaximierung zu Lasten des Gebührenzahlers“ angestrebt werde.

Michael Schmid-Ospach wird am 17. März vom Rundfunkrat von Radio Bremen (RB) zum neuen Intendanten und damit zum Nachfolger von Karl-Heinz Klostermeier gewählt. Schmid-Ospach verzichtet jedoch noch vor Amtsantritt aus gesundheitlichen Gründen auf das Amt. Klostermeier nimmt die Funktionen des Intendanten daraufhin ab dem 1. Mai kommissarisch wahr. Am 19. Juli wird Heinz Glässgen vom Rundfunkrat von Radio Bremen zum neuen Intendanten des Senders gewählt.

Die Intendanten des Saarländischen Rundfunks (SR) und des Südwestrundfunks (SWR), Fritz Raff und Peter Voß, unterzeichnen am 26. Januar einen Kooperationsvertrag, der eine Zusammenarbeit zwischen dem SR-Jugendradio „Unser Ding“ und dem digitalen SWR-Jugendradio „Das Ding“ vorsieht. Der SR will ab dem 1. März etwa sechs Stunden eigenproduziertes Programm in das Mantelprogramm von „Das Ding“ einfügen und im Saarland terrestrisch verbreiten.

Der Rundfunkrat des Westdeutschen Rundfunks stimmt einer Intensivierung der Fernsehregionalberichterstattung in den Ballungsräumen am 19. März zu. Ab 2000 soll in Köln und ab 2002 in Dortmund ein 50-minütiges Lokalprogramm im WDR-Fernsehen starten. Im Rahmen des sechsten WDR-Hörfunkprogramms Funkhaus Europa ist das 1995 gegründete gemeinsame Hörfunkmagazin „Radio E“ der Deutschen Welle sowie neun weiterer europäischer Auslandssender ab dem 9. Mai nicht mehr nur über Satellit, sondern auch über UKW zu empfangen.

Das ZDF startet am 9. Dezember seinen digitalen Theaterkanal. In zwei Programmblöcken soll das Filmmaterial von Aufführungen, Verfilmungen und Dokumentationen zugänglich gemacht und über das zeitgenössische Geschehen an deutschsprachigen Theatern informiert werden. Für den Empfang des Theaterkanals, der bundesweit in die Kabelnetze eingespeist wird und über Astra $1 \mathrm{G}$ zu empfangen ist, ist ein digitaler Decoder notwendig. 


\section{Privater Rundfunk}

\subsection{Landesmedienanstalten}

Die Gemeinsame Stelle Jugendschutz und Programm (GSJP) der Landesmedienanstalten beanstandet am 8. September eine Folge der US-Mystery-Serie „Charmed - Zauberhafte Hexen“ von Pro Sieben und eine Ausgabe der RTL-Talkshow „Bärbel Schäfer“ - „Alle hänseln mich, weil ich so hässlich bin“ - als jugendgefährdend.

Die Rundfunkkommission der Landesanstalt für Rundfunk Nordrhein-Westfalen (LfR) beschließt am 24. September die Zulassung von 17 digitalen TV-Programmen zur bundesweiten Verbreitung über Satellit und im Kabelnetz. Außerdem vergibt die LfR eine Lizenz zur bundesweiten Ausstrahlung eines griechischsprachigen Hörfunkprogramms über Satellit (Eutelsat Hotbird 13 Grad Ost). „Hellenic Radio Europe 1“ wird ein 24-Stunden-Programm mit Beiträgen aus den Bereichen Unterhaltung, Beratung, allgemeine und Kulturinformationen, Sendungen für griechische Schüler, griechische Popmusik und Sport anbieten.

Die Niedersächsische Landesmedienanstalt für privaten Rundfunk (NLM) erteilt den beiden Produktionsgesellschaften DCTP und Center TV am 29. September Lizenzen als unabhängige Dritte im Programm des Privatsenders RTL bis zum 21.7.2003.

Auf der Sitzung der Direktorenkonferenz der Landesmedienanstalten (DLM) am 25. Oktober in Dresden einigt sich die DLM mit der Telekom AG über die Nutzung der digitalen Kabelkanäle. Die einzelnen Landesmedienanstalten sollen die von der Telekom vorgeschlagene Belegung für die bisherigen 13 Digitalkanäle unter Berücksichtigung der landesrechtlichen Vorschriften übernehmen. Dem digitalen Pay-TV-Veranstalter Premiere World werden fünf komplette Kanäle zugewiesen, ARD und ZDF erhalten zusammen drei digitale Kanäle, ein Kanal ist für lokale und regionale Anbieter vorgesehen. Ein weiterer Digitalkanal wird das Telekom-Fremdsprachenpaket verbreiten mit ausländischen Fernsehsendern. Die privaten Fernsehsender RTL, SAT.1, Pro Sieben, RTL 2, Vox, Super RTL und tm3 sollen ebenfalls digital verbreitet werden, hierfür stehen allerdings bisher nur sechs Programmeinheiten zur Verfügung. Die DLM fordert daher von der Telekom AG die Schaffung weiterer Programmeinheiten auf den Sonderkanälen S33, S37 und S38.

Die niedersächsische Staatskanzlei teilt am 3. November mit, dass sich Niedersachsen nicht mehr an den Gesprächen über eine vereinigte norddeutsche Medienanstalt beteiligen werde, da die wirtschaftlichen Vorteile einer Fusion „eher marginal“ seien. Das Projekt erscheint daher endgültig als gescheitert.

Die Niedersächsische Landesmedienanstalt (NLM) erteilt der Anbietergemeinschaft Niedersachsen Rock 21 am 14. Dezember die Lizenz für die dritte UKW-Frequenzkette. Der Sender „Classic Rock“ (Zielgruppe 30- bis 60-Jährige) soll noch vor der Eröffnung der Expo-Weltausstellung am 1. Juni 2000 an den Start gehen.

Nach der KEK im Oktober stimmt am 15. Dezember auch die DLM der Lizenzierung eines türkischsprachigen TV-Vollprogramms der Dogan Media International $\mathrm{GmbH} z u$.

\subsection{Private Hörfunkveranstalter}

In Berlin geht am 7. Juni das türkischsprachige Radio 94,8 Metropol auf Sendung. Das 24-Stunden-Programm will neben türkischer Pop- und Unterhaltungsmusik etwa 25 Prozent der Sendezeit Service und Informationen bringen. 
Der Hörfunksender Rock Antenne München nimmt am 18. Oktober über eine digitale Astra-Satellitenfrequenz sowie über fünf Kabelfrequenzen in den Städten München, Ingolstadt, Augsburg, Regensburg und Nürnberg den Digitalradio-Regelbetrieb auf. Rock Antenne ist eine Tochter des bayerischen Privatradio-Anbieters Antenne Bayern.

Mit dem Jugendsender Sunshine Live geht am 21. November das erste landesweite Privatradio in Baden-Württemberg auf Sendung. Das Programm des Senders wurde bisher nur im Raum Nordbaden verbreitet und kann nun auch im restlichen Baden-Württemberg (mit Ausnahme des Südens und Nordostens) empfangen werden. Es ist serviceorientiert und lebt auch von den Höreranrufen, die zu jugendgemäßen Schwerpunktthemen in der Zeit von 14.00 bis $15.00 \mathrm{Uhr}$ gesendet werden.

\subsection{Private Fernsebveranstalter}

Rupert Murdoch erwirbt im Mai für seinen deutschen TV-Sender tm3 die Rechte an der Fußball-Champions-League ab September 1999. Murdoch ist mit 66 Prozent an tm3 beteiligt.

Der geplante Nachrichtensender N24 erhält von der Bayerischen Landeszentrale für neue Medien am 17. Juni eine bundesweite Sendegenehmigung. Die Kommission zur Ermittlung der Konzentration im Medienbereich (KEK) hatte am 18.5. grünes Licht für eine Zulassung des Sender, einer 100-prozentigen Tochter von Pro Sieben, gegeben. Der Nachrichtenkanal will im Januar 2000 auf Sendung gehen. Am 19. Oktober vereinbaren N24, der geplante Nachrichtensender der Pro-Sieben-Gruppe, und Bloomberg TV zunächst für drei Jahre - eine Kooperation, bei der Bloomberg TV täglich Live-Berichte von den Börsenplätzen Frankfurt am Main und New York zuliefern und zwei Talkformate für N24 produzieren wird.

Die Deutsche Telekom AG startet am 30. August auf der Internationalen Funkausstellung in Berlin ihre Vermarktungsplattform für digitale Kabelprogramme unter dem Namen Media Vision. Media Vision beinhaltet vier Pakete: Über Vision Basic können für DM 19,90 monatlich oder DM 238,- jährlich die Digital-TV-Programme von ARD Digital und ZDF Vision empfangen werden, Vision Select enthält einige Spartenprogramme, Vision Globe bietet kostenlos sechs fremdsprachige Programme für ausländische Mitbürger und in Vision Special werden ausländische Pay-TV-Programme, wie etwa das russische NTV, für bis zu DM 36,-- monatlich geboten. Zum Empfang der Programme ist eine Set-Top-Box mit der d-Box-Technik erforderlich.

Das digitale Pay-TV-Spartenprogramm Disney Channel erhält am 22. Juli die Zulassung zur bundesweiten Verbreitung vom Medienrat der Bayerischen Landeszentrale für neue Medien. Die Lizenz gilt für acht Jahre und ermöglicht den Sendestart des 24-stündigen deutschsprachigen Programms über die Plattform von Premiere World im Oktober.

Bei der Live-Übertragung des Schwergewichtsboxkampfes Schulz/Klitschko am 25. September und des Formel-1-Rennens am 26. September setzt $R T L$ die unzulässige Werbeform des Split-Screen-Verfahrens ein, bei dem das Fernsehbild in zwei Hälften unterteilt und gleichzeitig Werbung und Programm gesendet werden. Dies widerspricht zu diesem Zeitpunkt dem Rundfunkstaatsvertrag und den Werberichtlinien der Landesmedienanstalten. Nachdem die Niedersächsische Landesmedienanstalt für privaten Rundfunk (NLM) ein Beanstandungsverfahren gegen RTL eingeleitet hat, verzichtet RTL am 1. November vorerst auf diese Werbeform. Mit dem Inkrafttreten des novellierten Staatsvertrages am 1. April 2000 wird das Split-Screen-Verfahren gestattet, außer bei religiösen Sendungen und im Kinderprogramm. 
Die digitale Pay-TV-Plattform Premiere World startet am 1. Oktober. Sie ist entstanden aus der Fusion von Premiere und DF1, nachdem sich die Bertelsmann AG aus dem Pay-TV-Sender Premiere zurückgezogen hatte, und ist nun die einzige Pay-TV-Plattform in Deutschland. Eine Woche später gibt Premiere World den Abschluss eines Vertrages mit der Deutschen Netzmarketing GmbH (NMG) bekannt, der es den Kabelnetzbetreibern erlaubt, das Pay-TV mit zu vermarkten und dafür eigene Decoder einzusetzen, sofern diese ein piratensicheres Entschlüsselungssystem haben. Bisher ist der Empfang der digitalen Angebote von Premiere World nur mit der d-Box der KirchGruppe möglich.

Die Kommission zur Ermittlung der Konzentration im Medienbereich (KEK) teilt am 19. Oktober mit, dass der Zulassung des digitalen Programmbouquets RTL World keine Gründe der Sicherung der Meinungsvielfalt im Fernsehen entgegenstünden. RTL Television plant, seine Programme RTL, RTL 2, Super RTL sowie vier Spartenprogramme im Hyperbandbereich des Breitbandkabelnetzes als Bouquet weiterzuverbreiten.

In Nordrhein-Westfalen erhalten die „NRW 1 Privat TV GmbH“ und „NRW TV Fernsehen aus Nordrhein-Westfalen GmbH“ am 29. Oktober als erste Anbieter für ein landesweites 24-stündiges Satelliten-Vollprogramm eine Zulassung für 10 Jahre.

Die Ballungsraumsender Hamburg 1, TV Berlin, TV München, BTV Baden und BTV Württemberg, RNF plus (Rhein-Neckar Fernsehen), Saar TV, Sachsen TV und ein gutes Dutzend bayerischer Kleinst-Kanäle gründen am 12. November einen Verbund, der mit dem SAT.1-Vermarkter Media 1 zusammenarbeiten wird. Künftig wird es ein harmonisiertes Programmschema der Sender geben und SAT.1 wird Serien und sonntags ein TV-Movie zuliefern.

Die KEK gibt am 15. Dezember bekannt, dass sie keine konzentrationsrechtlichen Einwände gegen die Lizenzierung von sechs digitalen Spartenkanälen des Privatsenders tm3 erhebt. Die Kanäle sollen als Free-TV-Angebote über die Plattform von Premiere World ausgestrahlt werden. Auch für die Zulassung der digitalen TV-Spartenprogramme GSN Channel (Game Show Network) und SET Germany Channel der Sony Entertainment Television (SET) hat die KEK keine Bedenken. Die Programme sollen verschlüsselt bei Premiere World angeboten werden.

Quellen: epd medien, Funkkorrespondenz, Media Perspektiven, tendenz, wEv compact, Presseinformationen der Sender und Landesmedienanstalten, eigene Recherchen 